\title{
Efficacy of Traditional Chinese Medicine On 2019-Ncov: Two Case Reports
}

\section{ZZ Zheng ${ }^{1}$, NN Ma ${ }^{2}, \mathrm{~L} \mathrm{Li}^{3}$, Dan Jiang ${ }^{4 *}$}

${ }^{1}$ TCM Consultant, Special Appointed Hospital for nCoV, Jiangxia District TCM hospital, Wuhan, PR China.

${ }^{2}$ Doctor of TCM, Special Appointed Hospital for nCoV, Jiangxia District TCM hospital, Wuhan, PR China

${ }^{3}$ Imperial College, London, UK

${ }^{4}$ TCM Consultant, Hallam Institute of TCM, Sheffield, UK.

*Corresponding author: Dan Jiang, TCM Consultant, Hallam Institute of TCM, Sheffield, UK.

Received Date: February 17, 2020; Accepted Date: February 20, 2020; Published Date: March 11, 2020.

Citation: Zheng ZZ, Ma NN, Li L, And Jiang D. (2020) Efficacy of Traditional Chinese Medicine On 2019-Ncov: Two Case Reports. Journal of Clinical Case Reports and Studies, 1(1): 10.31579/ 2690-8808/005

Copyright: ( ) 2020. Dan Jiang. This is an open-access article distributed under the terms of the Creative Commons Attribution License, which permits unrestricted use, distribution, and reproduction in any medium, provided the original author and source are credited.

Novel coronavirus (2019-nCoV)-infected pneumonia (NCIP) is a new viral illness initially identified in the central Chinese city of Wuhan in December 2019. According to the daily report on the epidemic situation of $\mathrm{nCoV}$ issued by the National Health and Family Planning Commission of the People's Republic of China on 14 February 2020: 2019-nCoV is highly infectious, causing extremely high incidence of NCIP throughout Wuhan, and has spread swiftly to 34 provinces within China and 24 other countries around the world between January and February 2020. There have been 63,946 cases diagnosed as nCov pneumonia; 10,109 suspected cases, and 1,382 deaths in China (mortality rate of $4.05 \%$ ). Outside China there have also been 515 confirmed cases and two deaths (1). The epidemic and clinical analyses to NCIP published by the Chinese Centre for Disease Control and prevention in the New England Journal of Medicine (2) and in JAMA by the medical teams in Wuhan (3) in Feb 2020. As NCIP is a novel viral disease, there is no extant successful treatment model in conventional modern medicine. As the morbidity and mortality rates for SARS (Severe Acute Respiratory Syndrome) had decreased after the successful introduction of Traditional Chinese herbal medicine (TCM) during its outbreak in 2003 (4-6), and following our experience in treating viral illness with TCM, we have been applying
Chinese Herbal medicine to treat patients with NCIP since January 2020 and have gained satisfactory results. We report two successful cases here.

\section{Case 1}

Ms Zhu, 38 year old female, resident of Wuhan, presented with fever and rigors, dry cough and dry mouth without sputum, dizziness, aching limbs, depressive mood, and anorexia. She had been self-medicating (drug unknown) for three days prior to presentation, but had felt no improvement. Chest CT showed multiple infective speckles and flakes in both lungs. She was diagnosed with pneumonia and admitted to hospital. During her stay on a general medical ward, she was commenced on ceftazidime antibiotic and a patent Chinese herb infusion (Xiyan Ping) for four days, but showed no significant improvement. A PCR (Reverse Transcription-Polymerase Chain Reaction) diagnosis of $\mathrm{nCoV}$ pneumonia was subsequently made.

The patient was then transferred to our department with a fever of $39.2 \mathrm{C}$, slight aversion to cold, bitter taste and dry mouth, dry cough without sputum, dizziness, aching limbs, and anorexia. She was found to have a red tongue with thin-yellow-greasy coating. Chest CT is displayed as below:

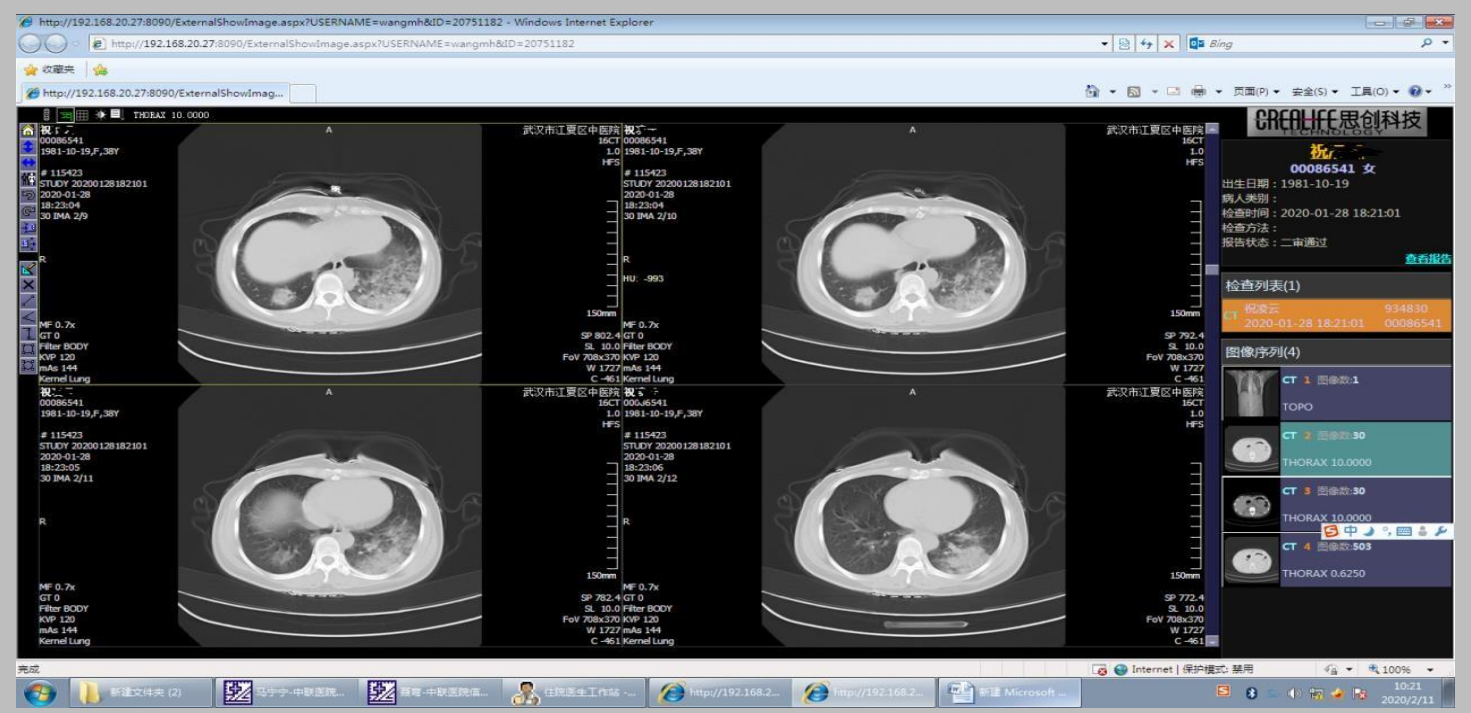


During admission, she received in addition to ceftazifime and Xiyan Ping, an herbal formula targeted to her symptoms above.

TCM diagnosis: combinative pattern of larger yang meridian and lesser yang meridian

Modification of mixed of Xiao Chaihu and Maxingshigan decoctions

Prescription:

Chaihu (Radix Bupleuri) 20,

Huangqin (Radix Scutellariae) 12,

Fabanxia (Rhizoma Pinelliae) 12,

Dangshen (Radix Codonopsis Pilosulae) 15

Ganjiang (Rhizoma Zingiberis) 10,

Hongzao (Fructus Zizyphi Jujubae) 12,

Zhigancao (Radix Glycyrrhizae) 10,

Mahuang (Herba Ephedrae) 10
Xingren (Semen Armeniacae) 12,

higao (Gypsum Fibrosum)30,

Lugen (Rhizoma Phragmitis) 30,

Ziyuan (Radix Asteris) 15

Kuandonghua (Flos Farfarae) 15,

Chantui (Periostracum Cicadae) 10

Yiyiren (Semen Coicis) 20,

Maiya (Fructus Hordei Germinatus) 20

Above herbal mixture was decocted with warm water and taken orally twice daily.

Sweating and temperature decreased after taking a dose of herbs; she reported resolution of most of her symptoms after three doses, and her PCR test became negative. Her temperature changes are displayed below:

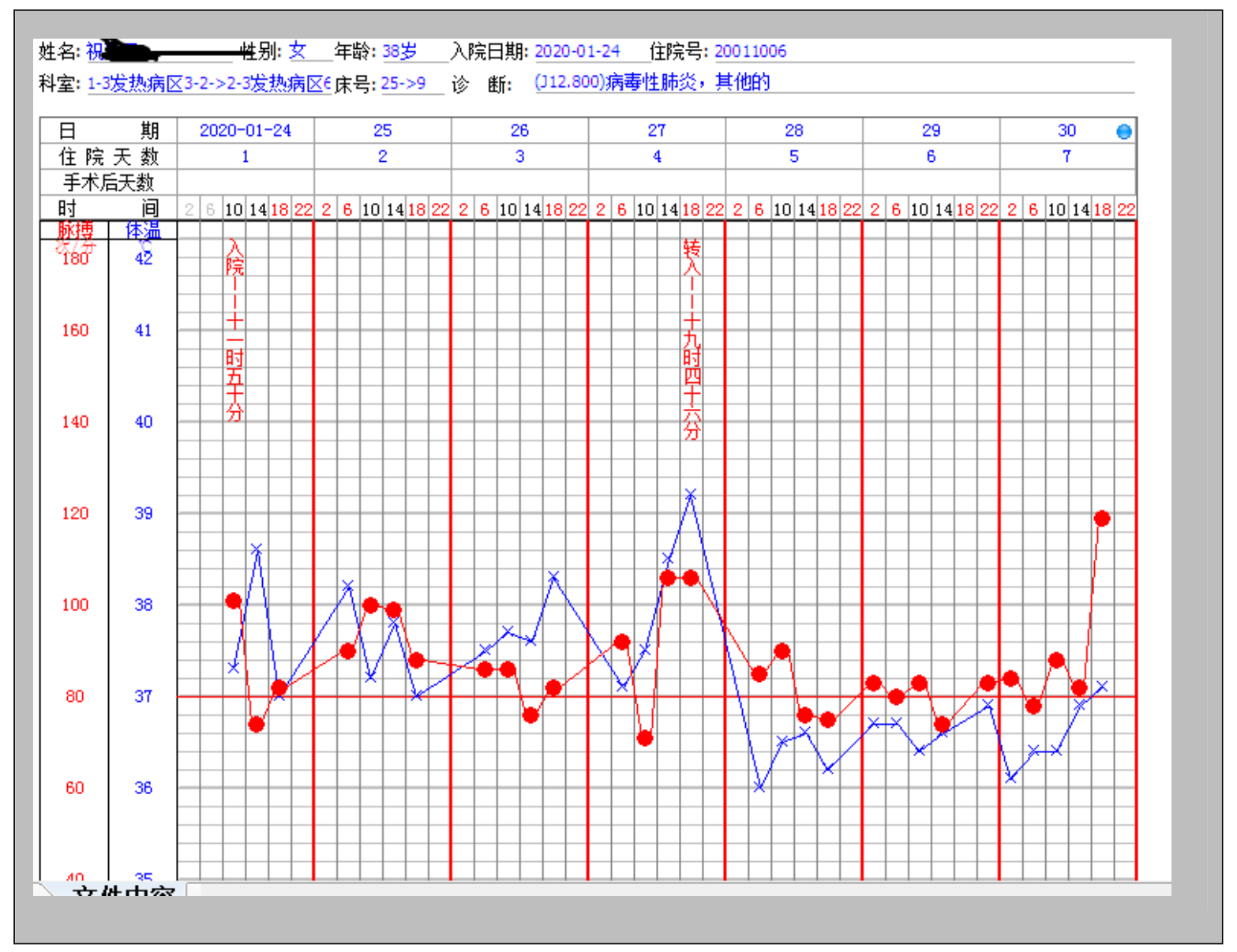

Temperature chart 1: 


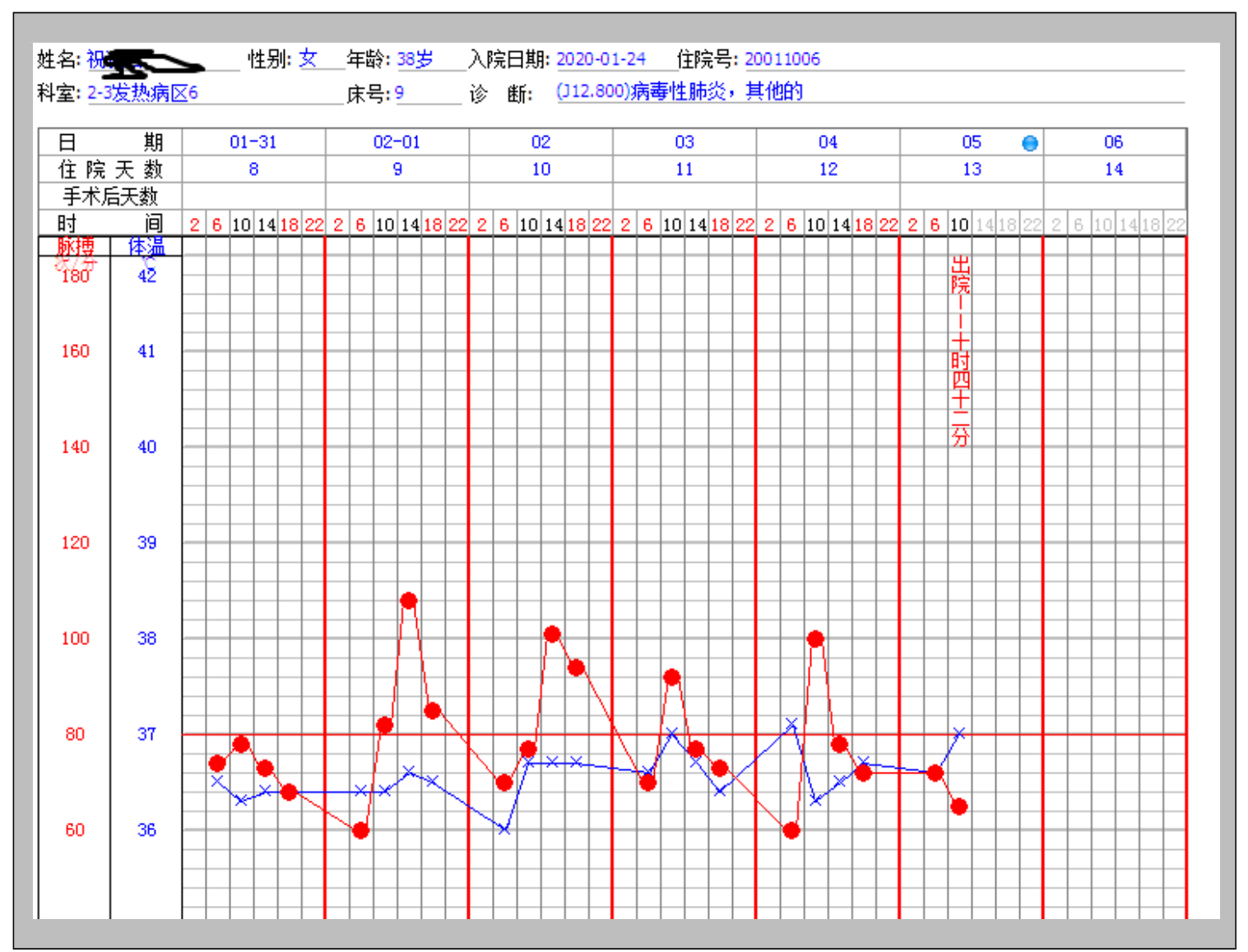

Temperature chart 2:

Note:

- $\quad$ Red dots represent heart rate;

- $\quad$ Blue crosses represent temperature;

- The first chart is recorded from the first week of her admission $\left(24^{\text {th }}\right.$ to $30^{\text {th }}$

Jan), the secondary chart is recorded in the $2^{\text {nd }}$ week ( 31 st Jan to $5^{\text {th }} \mathrm{Feb}$ ). The patient was discharged on $6^{\text {th }}$ Feb 2020.
The TCM prescription was not altered any further as the patient improved. After completing her course of herbs, repeat PCR testing was negative for $\mathrm{nCoV}$. Repeat CT scan showed reduction but not complete disappearance of the typical ground glass appearance, and so herbs were continued for a further five days. The patient improved daily without recurrence of symptoms. 


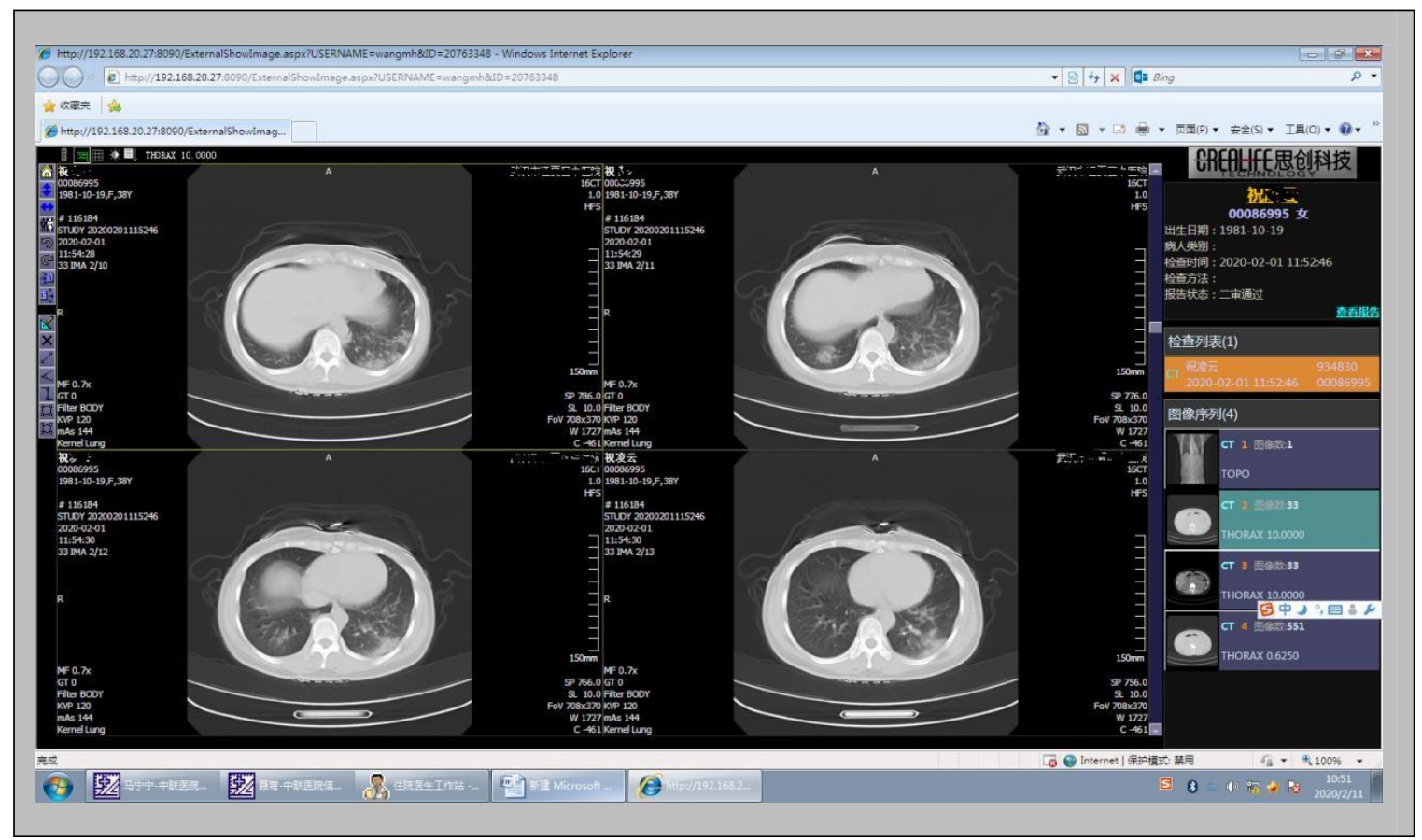

Chest CT 2:

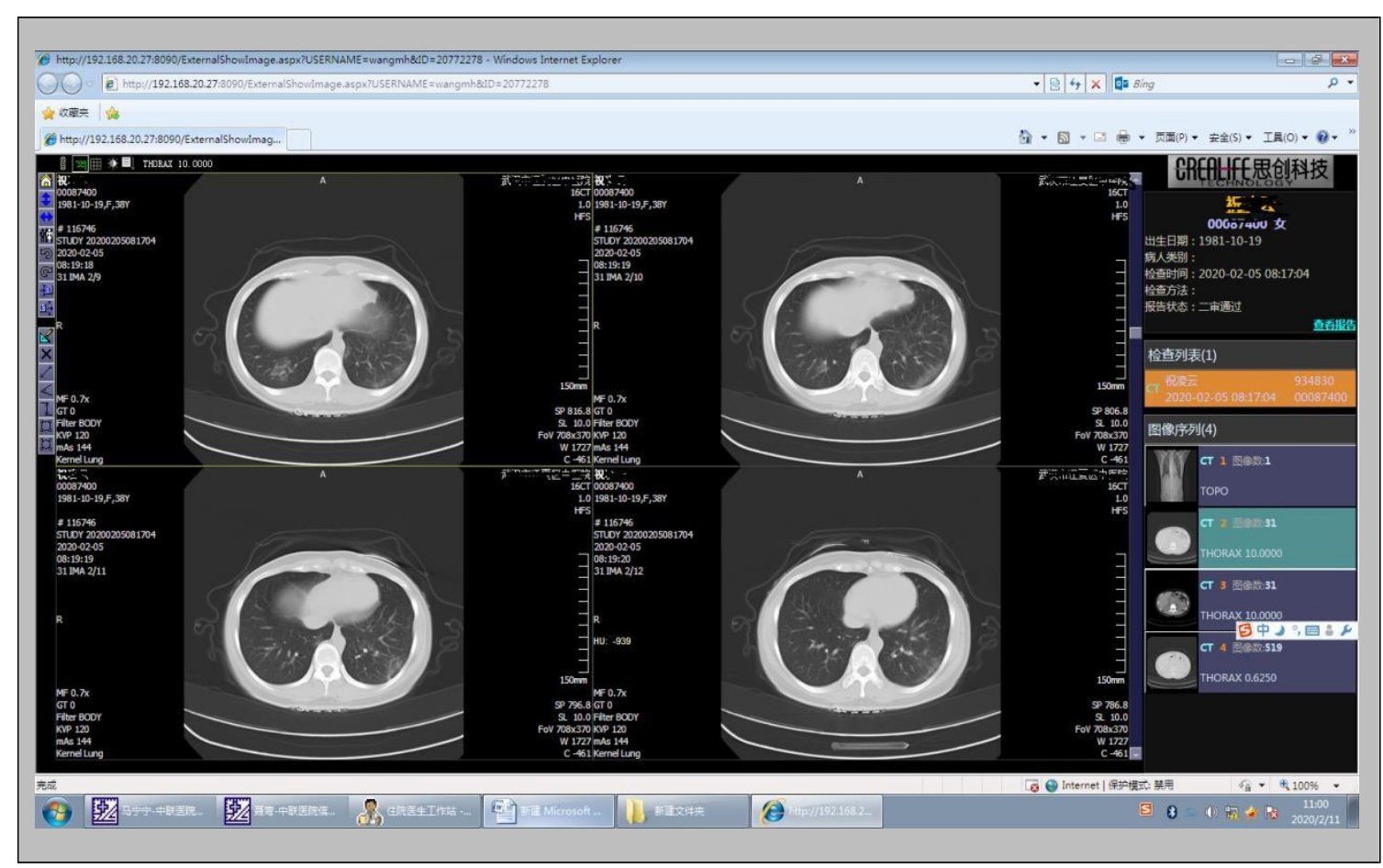

\section{Chest CT 3}

A third CT scan showed almost complete disappearance of the ground glass appearance. On discharge the patient had complete resolution of symptoms.

\section{Case 2}

$\mathrm{Mr} \mathrm{Li}, 60$ year old male, resident of Wuhan, presented to the district hospital with fever and rigors, dry cough, aching muscles, breathlessness

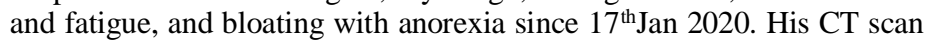
(below) showed an infective appearance in both lungs, and a diagnosis of viral pneumonia was suspected. He received unknown out-patient treatment. 
The patient did not improve after initial treatment. He was admitted on $24^{\text {th }}$ Jan 2020 to a general ward and commenced on ceftazidime and moxifloxacin, oseltamivir, anti-influenza patent herbs (Lianhua Qingwen capsules and Xiyanping infusion), and ibuprofen as a baseline treatment. Due to ongoing fever above 40C and positive PCR testing, he was admitted to the special nCoV ward.

On admission to our unit, he appeared in poor spirits with intermittent fevers in the afternoons reaching 40C, headache, general muscular aching, dry cough, breathlessness and fatigue, activity-induced wheeze, bloating and anorexia. His tongue was red with yellow-thick-greasy coating;

TCM diagnosis: dampness and heat accumulation

Treatment principle: to release excessive dampness and clear accumulated heat

Herbal prescription: Sanren Decoction modification

Xingren (Semen Armeniacae) 10,

Baidoukou (Fructus Amomi Cardamomi) 10,

Yiyiren (Semen Coicis) 30
Houpo (Cortex Magnoliae Officinalis) 10,

Fabanxia (Rhizoma Pinelliae) 10,

Tongcao (Medulla Tetrapanacis) 10,

Gancao (Radix Glycyrrhizae) 10,

Huashi (Talcum) 10,

Zhimu (Rhizoma Anemarrhenae) 10

Huangqin (Radix Scutellariae) 10,

Mahuang (Herba Ephedra) 8,

Fuling (Poria) 10

Chaihu (Radix Bupleuri) 10,

Zhuye (Folium Bambusae) 10

The above herbal mixture was decocted and taken orally as herbal juice twice daily for five days.

\section{Result}

Fever and sweats decreased after one dose of the above herbs. All symptoms reduced after three doses. His temperature is seen in the chart below:

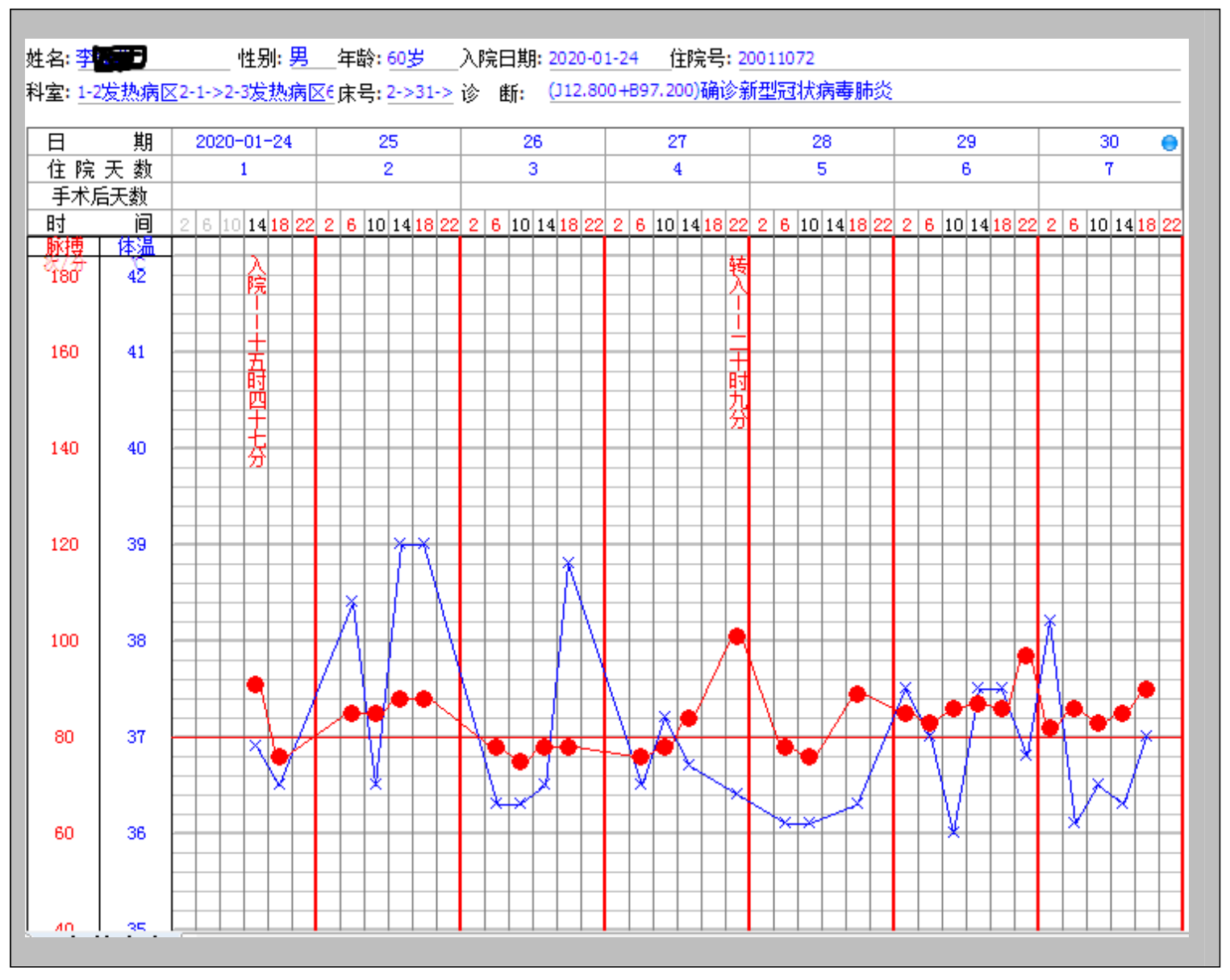

Temperature chart 1: 


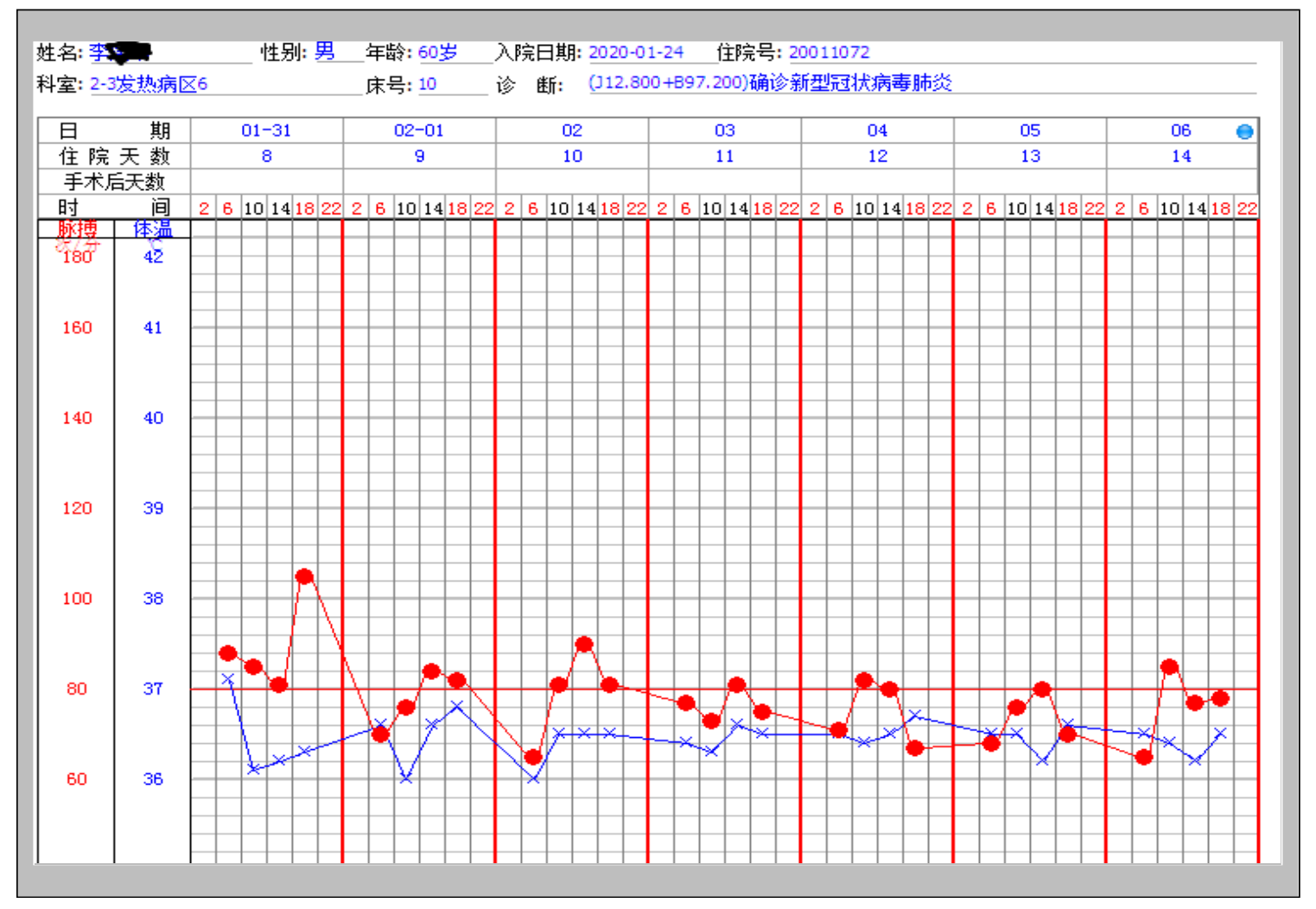

Temperature chart 2:

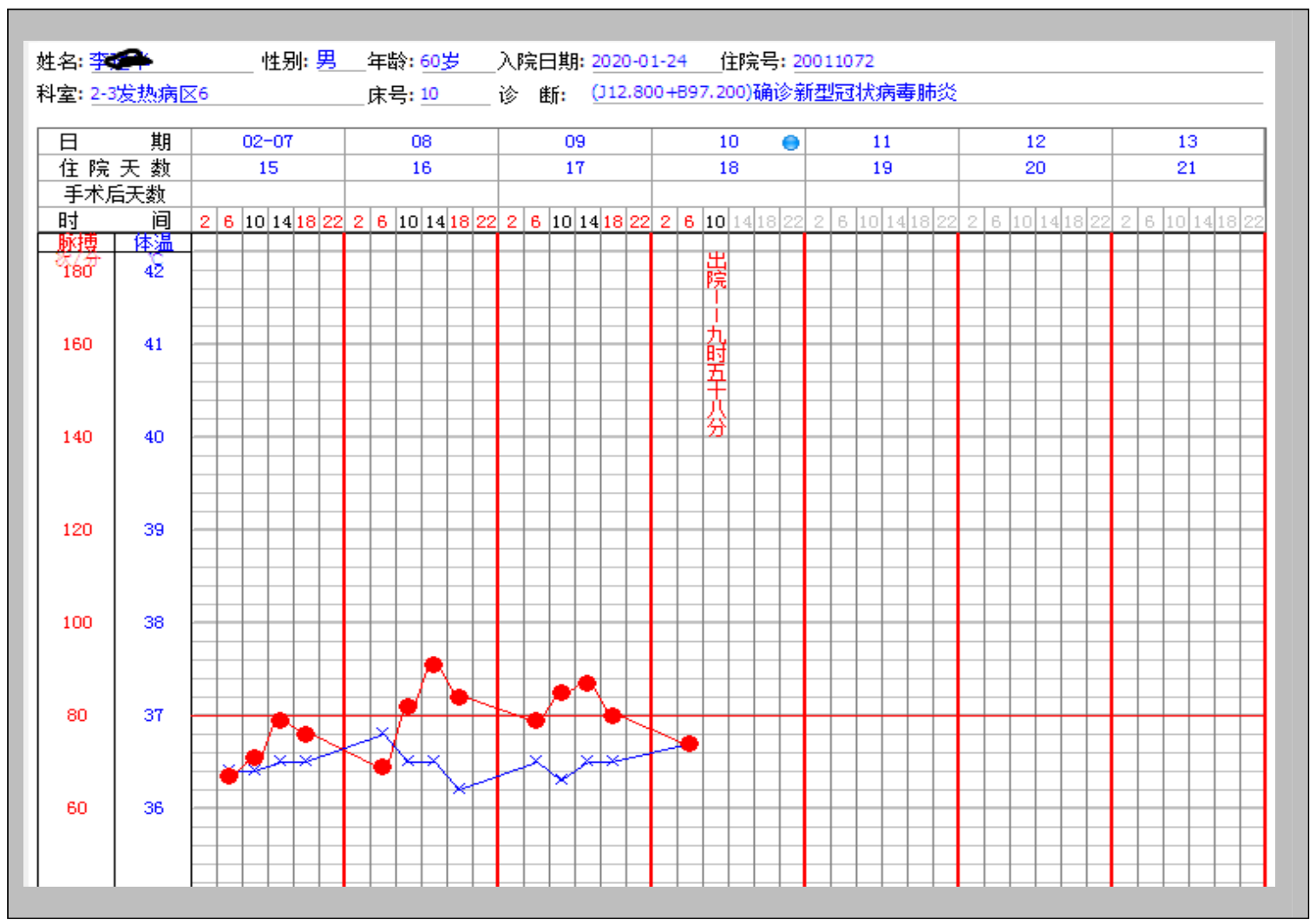

Temperature chart 3: 
Note:

- $\quad$ Red dots represent heart rate;

- $\quad$ Blue crosses represent temperature;

- Chart 1 is recorded for the first week after his admission; chart 2 for the Second week; and the chart 3 for the third week.

After five days, the patient reported feeling less breathless and bloated with increased appetite, but still complained of general muscular aching. The herbal

Prescription was modified on $28^{\text {th }}$ Jan as below:
Zhimu and Huangqin are removed;

Qianghuo (Radix ET Rhizoma Notopterygii) 10

Gegen (Radix Puerariae) 10

Mugua (Fructus Chaenomelis) 10

Binlang (Semen Arecae) 10

Shanzha (Fructus Crataegi) 10

Shenqu (Massa Fermentata Medicinalis) 10 added

The above herbal mixture was prescribed for seven doses. The patient reported feeling much less muscle ache after one dose of the new prescription, and his PCR testing also returned negative at this point. His chest CTs are seen as below:
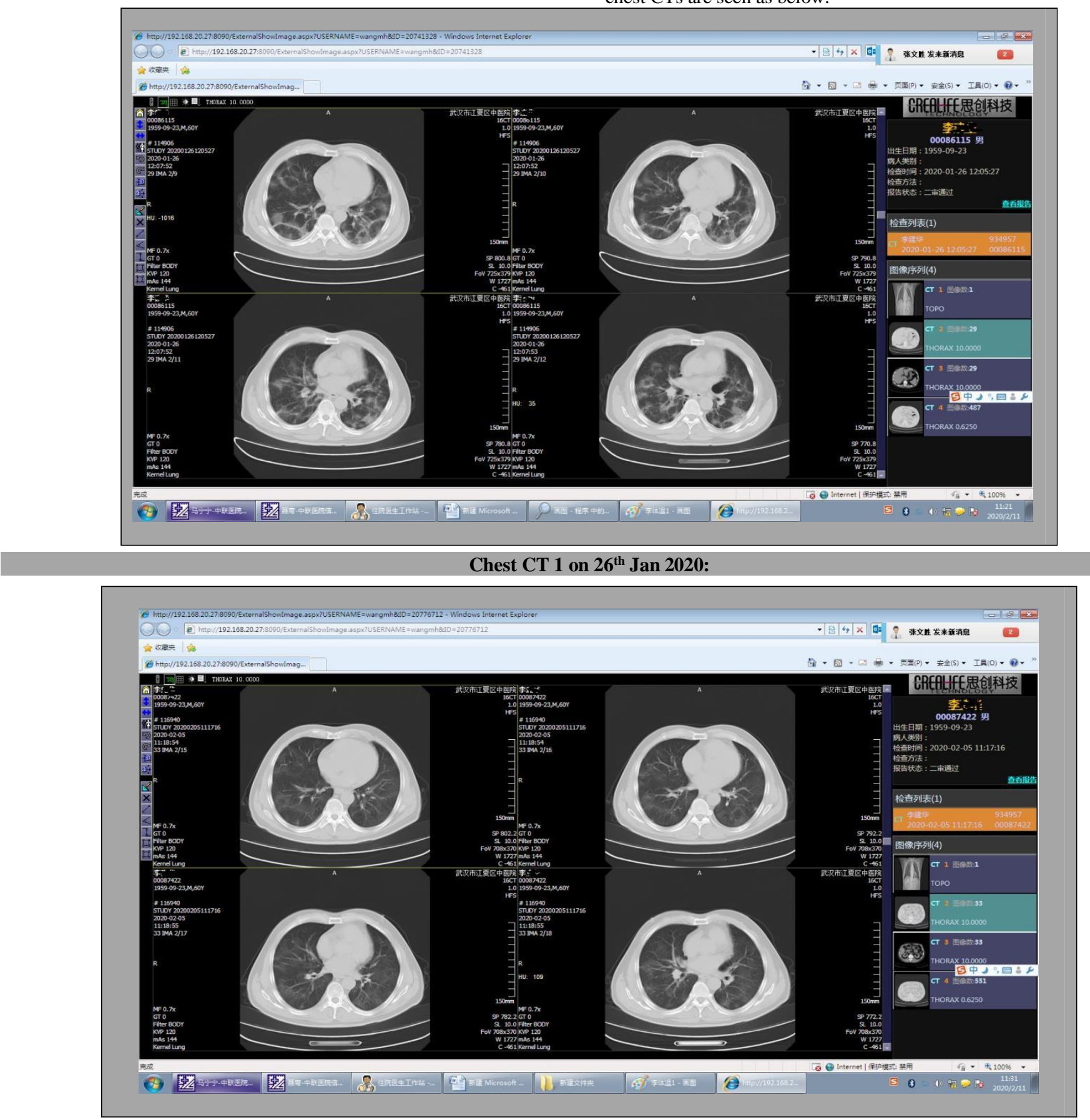

Chest CT 2 on $5^{\text {th }}$ Feb 2020: 


\section{PCR was negative on $8^{\text {th }}$ Feb 2020.}

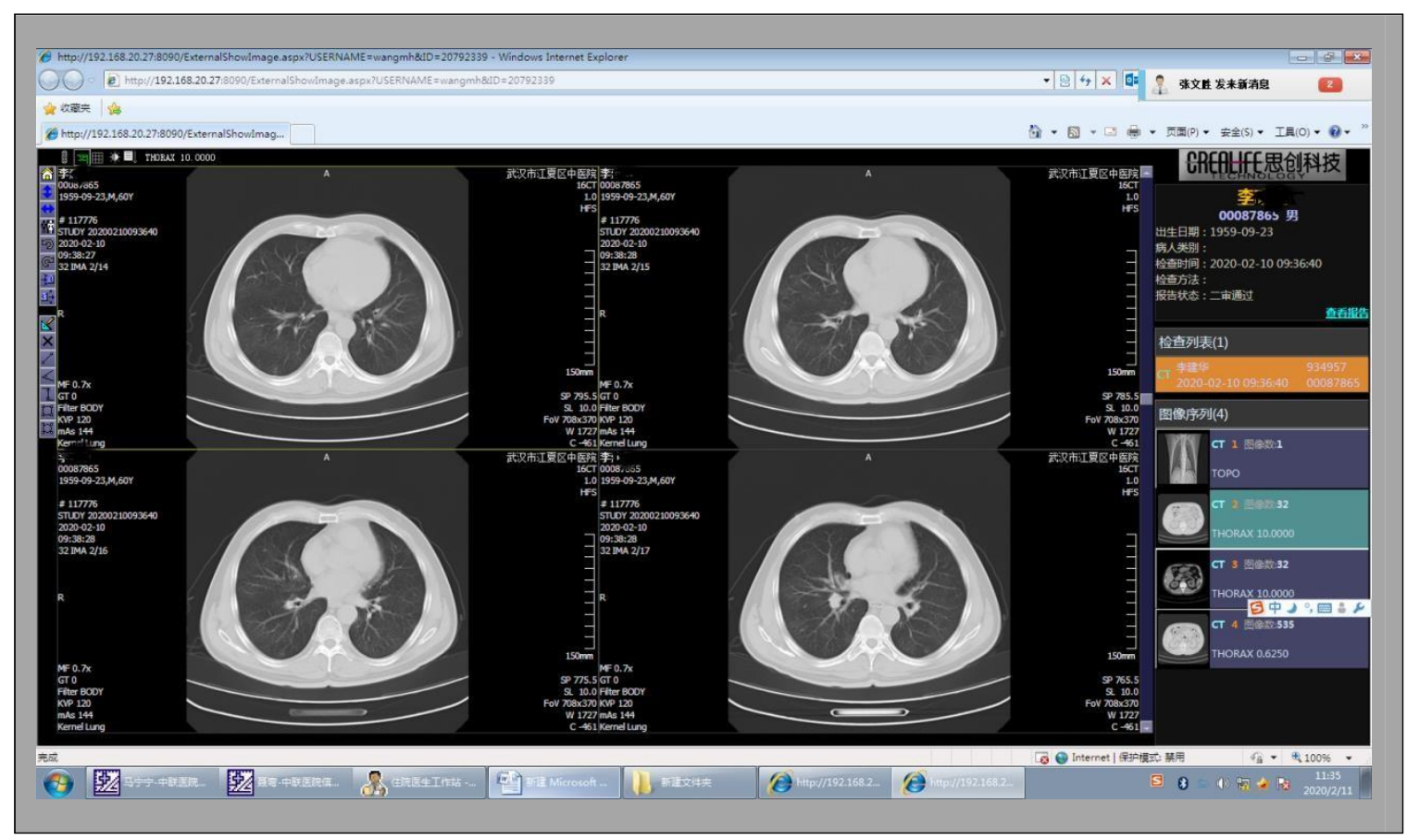

Chest CT 3 on $10^{\text {th }}$ Feb 2020:

The patient was discharged with complete clinical and laboratory resolution, and almost complete radiological resolution on $10^{\text {th }} \mathrm{Feb} 2020$.

\section{Summary}

NCIP is an infectious disease caused by a novel virus which possesses strong person-to-person transmission. It has been widespread throughout Wuhan, China since January 2020, with a mortality rate of $4.3 \%$, which is much higher than that of the common influenza virus, $0.2 \%(3,7)$. To date, there is no effective method to control this virus by the conventional modern medicine. We have preliminarily gained satisfactory treatment outcomes when we have applied traditional Chinese herbal medicine to patients suffering from NCIP. Our experience can be summarized in the following points:

1. Most of patients have already received routine conventional western medicine

Treatment as out-patients or on general medical wards before admission to the special nCoV ward (such as antibiotics and antivirals). Despite initial routine treatment, NCIP patients go on to develop more severe symptoms and demonstrate worsening CT appearances. During admission, special herbal prescriptions tailored to the particular patient has been shown to be effective at controlling symptoms and resolving radiological and laboratory markers.

2 TCM pays more attention to the patient's individual characteristics and different stages of disease to prepare the specialised prescription. The general correction of a patient produced by herbal medicine may regulate and repair the impaired immune function caused by NCIP and may be the key to promoting NCIP disease resolution.

3 Due to the lower cost and side-effect profile of herbs, we can also shorten the treatment period and healthcare costs for the patient.

Due to such positive results from TCM, in our unit we have incorporated herbal medicine into the routine treatment regime for patients with NCIP. Due to the novel nature of NCIP, literature searches have returned few results. We have identified one other case report with a similar experience to ours: the authors applied an integrative approach of conventional western medicine plus traditional Chinese medicine to treat NCIP and compared results with applying conventional medicine only. Their results showed that the integrative approach decreased times to resolution of symptoms, to fever-clearance, and to length of hospital stay; conversely the integrative approach increased the rates of clinical and radiological improvement, it also reduced the proportion of severe cases ( 8 and figure below). 


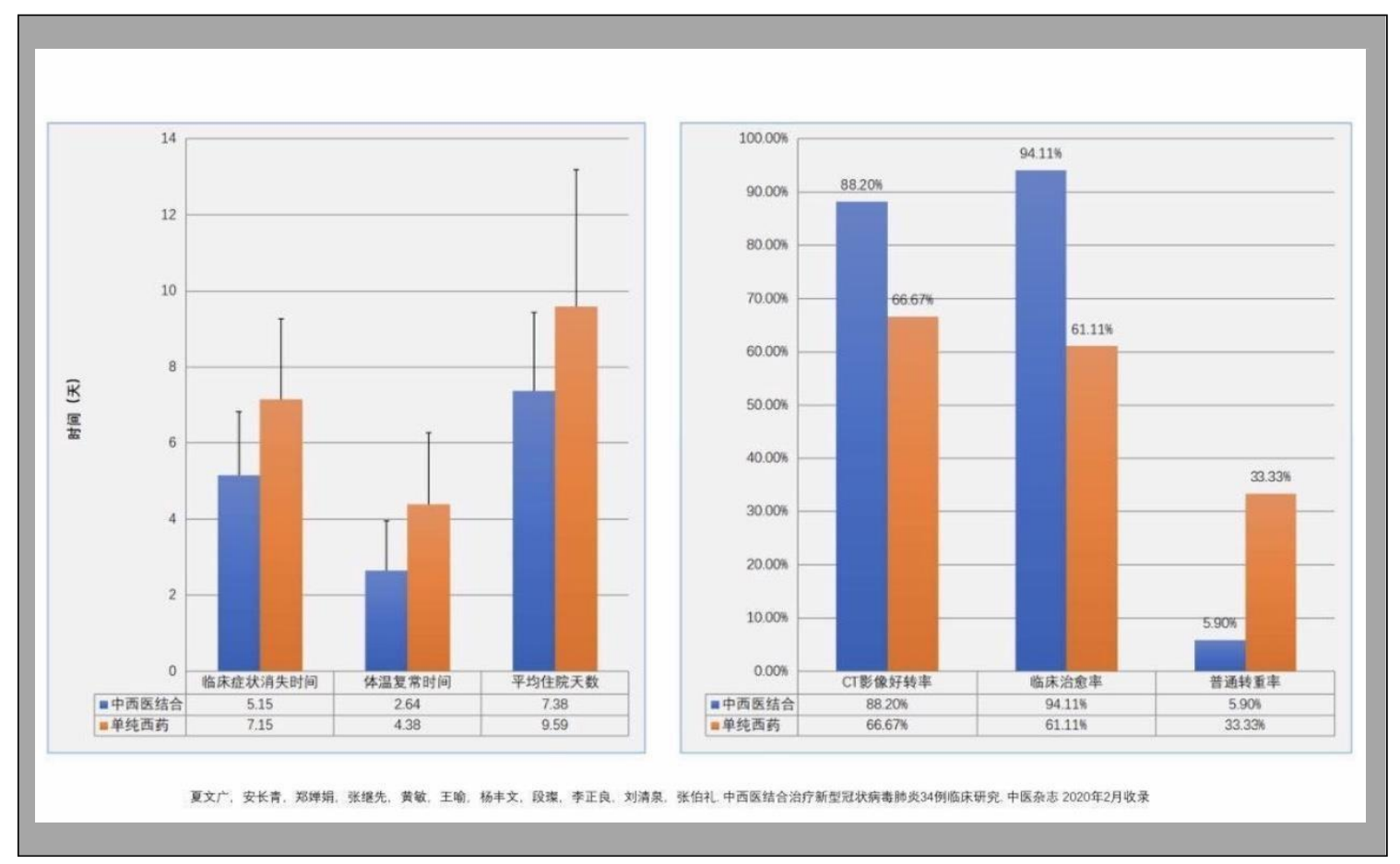

The efficacy of results such as ours has been reflected in the latest treatment guideline issued by the National Health and Family Planning Commission of PRC. Its fifth edition guidelines have now incorporated Chinese herbal medicine into its recommendations for treating NCIP (9). Further reports of our results with TCM are planned with larger cohorts.

\section{References}

National health and Family Planning Commission of the People's Republic Of China " the epidemic situation on nCoV" '14 ${ }^{\text {th }}$ Feb2020

1. Zhu N, Zhang D, Wang W, Li X, Yang B, Song J et al ( 2020) ) A Novel Coronavirus from Patients with Pneumonia in China, 2019; New England Journal of Medicine.

2. Wang D, Hu B, Hu C, Zhu F, Liu X, Zhang J, et al (2020) Clinical Characteristics of 138 Hospitalized Patients With 2019 Novel Coronavirus-Infected Pneumonia in Wuhan, China. Journal of America Medical Association.

3. Zhao CH, Guo YB, Wu H, Li XH, Guo XH, Jin RH et al (2003) Clinical manifestation, treatment, and outcome of severe acute respiratory syndrome: analysis of 108 cases in Beijing; Chinese Medical Journal; 83 (11):897-901. PMID: 12899786
Lau JT, Leung PC, Wong EL, Fong C, Cheng KF, Zhang SC, et al (2005) the use of an herbal formula by hospital care workers during the severe acute respiratory syndrome epidemic in Hong Kong to prevent severe acute respiratory syndrome transmission, relieve influenza-related symptoms, and improve quality of life: a prospective cohort study. Journal of Alternative and Complementary Medicine. 11(1): 49-55.

5. Liu BY, He LY, Liang ZW, Tong XY, Hu JQ, Jiao Q, et al (2005) Effect of glucocorticoid with traditional Chinese medicine in severe acute aspiratory syndrome (SARS)]. China Journal of Chinese Materia Madica 30(23):1874-7.

6. www.chyxx.comm Market and Space analysis on Chinese Incidence, Mortality and prevention to Influenza in 2018-2019, April 2019

7. GW Xia, CQAn, CJ Zheng, JX Zhang, QQ Liu, Bl Zhang et al (2020) Clinical study on integrative medicine treat $\mathrm{nCoV}$ pneumonia with 34 cases study; Journal of Chinese Medicine 2

8. National Health and Family Planning Commission of PRC (2020): Treating Guides on nCoV in the fifth issue.

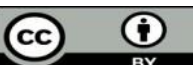

This work is licensed under Creative Commons Attribution 4.0 License

\section{To Submit Your Article Click Here: Submit Manuscript}

DOI: $10.31579 /$ CCRS.2020/005

4.
Ready to submit your research? Choose Auctores and benefit from:

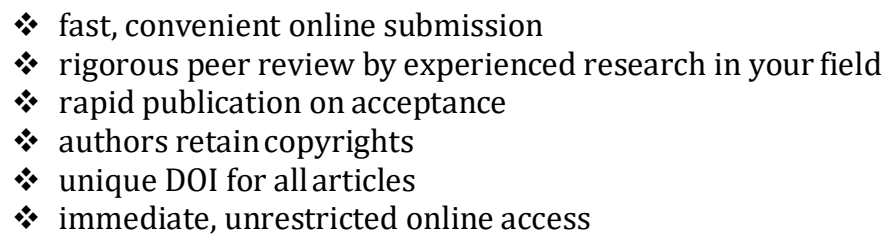

At Auctores, research is always in progress.

Learn more www.auctoresonline.org/journals/clinical-case-reports-andstudies 